\title{
A novel double threshold-based spectrum sensing technique at low SNR under noise uncertainty for Cognitive Radio Systems
}

Garima Mahendru ( $\sim$ gmahendru@amity.edu )

Amity University https://orcid.org/0000-0002-5484-3623

\section{Research Article}

Keywords: Spectrum measurement, energy detection, noise uncertainty, dynamic threshold, threshold wall, probability of detection, probability of error

Posted Date: April 15th, 2021

DOI: https://doi.org/10.21203/rs.3.rs-397280/v1

License: (a) (i) This work is licensed under a Creative Commons Attribution 4.0 International License.

Read Full License 


\title{
A novel double threshold-based spectrum sensing technique at low SNR under noise uncertainty for Cognitive Radio Systems
}

\author{
Garima Mahendru \\ Amity University Uttar Pradesh \\ gamahendru@amity.edu
}

\begin{abstract}
Cognitive Radio is a novel concept that has invoked a paradigm shift in wireless communication and promises to solve the problem of spectrum underutilization. Spectrum sensing plays a pivotal role in a cognitive radio system by detecting the vacant spectrum for establishing a communication link. For any spectrum sensing method, detection probability and error probability portray a significant part in quantifying the detection performance. At low SNR, it becomes cumbersome to differentiate noise and signal due to which sensing method loses robustness and reliability. In this paper, mathematical modeling and critical measurement of detection probabilities has been done for energy detection-based spectrum sensing at low SNR in uncertain noisy environment. A mathematical model has been proposed to compute double thresholds for reliable sensing when the observed energy is less than the uncertainty in the noise power. A novel parameter "Threshold Wall" has been formulated for optimum threshold selection to overcome sensing failure. Comparative simulation and analytical result measurements have been presented that reveals improved sensing performance.
\end{abstract}

Index Terms--Spectrum measurement, energy detection, noise uncertainty, dynamic threshold, threshold wall, probability of detection, probability of error.

\section{INTRODUCTION}

In the present pandemic situation due to the spread of COVID 19 virus worldwide, where millions of people are forced to stay at home with schools/ colleges/ offices/ entertainment theatres closed, people are now spending more of their times online. The internet usage and its consumption has surged significantly and has pushed people of all ages to online mode for work, play and education. With the rise in social distancing norms, users are seeking new ways to connect mostly through internet according to the recent collated reports from telecom industry [1].

The main problem encountered while rendering such wireless services is the spectrum scarcity. Research studies carried out by FCC state that the use of fixed spectrum allocation policies makes the wireless spectrum underutilized [2]. The measurement of spectrum usage portrays the underutilization as well as overcrowding of few chunks of RF spectrum [3-4]. The reason behind spectrum scarcity is the inefficient existing scheme of spectrum allocation. In the present situation, the spectrum scarcity problem has aggravated and needs attention. The issue of spectrum crunch is of utmost significance and needs immediate action for future networks.

For efficient spectrum usage, un-licensed secondary users (SU) can share and access the spectrum along with licensed primary users (PU) with the help of Dynamic Spectrum Allocation via Cognitive Radio [5]. Cognitive Radio (CR) has invoked a paradigm shift in wireless communication era and has the intelligence to detect the vacant band of spectrum and adapt its parameters accordingly [6-7]. For cognitive radio systems, identification of spectrum availability in the vicinity is the most crucial task which is implemented through spectrum sensing. For spectrum sensing in CR the primary objective is to achieve the desired Receiver Operator Characteristics (ROC) in terms of detection probability $\mathrm{P}_{\mathrm{D}}$, false alarm probability $\mathrm{P}_{\mathrm{FA}}$, number of samples $\mathrm{N}_{\mathrm{s}}$, sensing duration at an acceptable level of SNR (Signal to noise ratio).

The main contribution of this work is given as follows: firstly, a novel mathematical framework for computing the "Threshold Wall" has been proposed and simulated to overcome a sensing failure without additional hardware or detection stage. To further enhance the system performance a novel concept of adaptive double threshold has been proposed to make the sensing reliable. The variation in the adaptive thresholds with respect to noise uncertainty has been quantified and formulated as the "Threshold Wall" to avoid sensing error at low SNR in presence of noise uncertainty. The computation of double thresholds prevents 
detection malfunction and hence reduces the overall error probabilities. This work presents a mathematical model to compute double thresholds for reliable sensing and a new constraint to avoid failure with "Threshold Wall".

The rest of the paper is organized as: Section 1.1 describes the recent related work in the field of spectrum sensing in cognitive radio networks. Section 2 presents the conventional system model in a spectrum measurement process and the exhibits the shortcomings as well. Section 3 proposes a novel method of spectrum sensing through adaptive double thresholds. Section 4 gives the simulation results and presents a comparative analysis of the proposed technique with the existing ones. The paper has been summarized and concluded in section 5 .

\subsection{Related Work}

Over two decades, researchers worldwide explored upon various methods for spectrum sensing. Based on the literature survey on spectrum sensing various methods like: Energy detection (ED) [8-11]; Cyclo-stationary feature detection [12-14]; Matched filter detection [15-16]; Statistical covariance based detection [17-18]; Cooperative sensing [19-20]; compressed sensing [21-23] were proposed to examine the spectrum occupancy under different scenarios. Considering the research on spectrum sensing methods; energy detection draws the attention of the researchers for its lesser computational and operational complexity [24]. Energy Detection doesn't require a priori knowledge about the primary user (PU) signal being sensed but is significantly affected by noise conditions [25]. The major constraint that puts a check on the performance of the energy detection (ED) method is selection of threshold at low SNR (signal-to-noise-ratio). Fixed single threshold concept at low SNR prolongs the sensing time. The situation worsens with flickering noise power and leads to sensing failure below a certain SNR (termed as "SNR wall") which is characterized by low $\mathrm{P}_{\mathrm{D}}$ and increased probability of error or error rates The noise power fluctuation causes degradation in the performance of a spectrum sensing method which can be improved by using dynamic threshold factor [26-28]. The authors of [29] have presented a double threshold-based sensing technique that narrows down the uncertainty zone to increase the robustness of the system against noise power fluctuations. Several adaptive double threshold-based detection methods have been proposed which incorporates an adaptive factor in computation of the decision threshold to improve the robustness of the system against noise [30-32]. The authors in [33] have proposed a two-stage scheme, fitted to perform in TV bands. It promises to offer a good detection of the occupied bands with desirable false alarm rates (Stage 1) and an exact measurement of the real occupied spectrum (Stage 2). A new adaptive sensing time technique-based spectrum sensing that depends on the SNR has been given in [34] to increase the literal spectrum usage and the achievable throughput. Apart from using double threshold, some methods employ machine learning or optimization algorithms to achieve desirable sensing performance and prevent sensing failure [3536]. Authors in [37] have proposed the use of artificial neural networks (ANN) to implement hybrid spectrum sensing. However, all these methods require extra implementation cost and increased hardware complexity. Our previous work in [38] aimed at introducing a novel mathematical model for energy detection-based sensing. It was re-modeling of the existing mathematical system to achieve desirable performance metrics without changing the sensing algorithm.

All the methods discussed above, aim at reducing the error probability or increasing the detection probability as well as throughput. None of the methods, aimed at inclusion of a minimum threshold value that should be taken into consideration at low SNR. Moreover, adaptive double threshold not only divides the sensing scenario in two regions of high and low SNR but also varies it according to the changing SNR.

Therefore, this paper aims at spectrum measurement at low SNR in the presence of uncertainty in noise with the help of double thresholds that can adapt its value according to the varying noise. The measurements of the detection probability and error rate through the simulation depicts improvement as well as reduction in the width of uncertainty zone at low SNR.

\section{SYSTEM MODEL}

Spectrum sensing via energy detector involves observation of received signal energy over a certain time interval [7]. Signal detection is tested by formulation of two hypotheses with the following conditions:

$$
\left\{\begin{array}{c}
\boldsymbol{H}_{\mathbf{0}}: X(n)=N(n) \\
\boldsymbol{H}_{\mathbf{1}}: X(n)=S(n)+N(n)
\end{array}\right\}
$$

where $X(n)$ is the received signal samples at CR node, $S(n)$ is the primary signal of interest and $N(n)$ is the noise samples. Assume that the noise is Gaussian with zero mean and band limited power spectral density. $H_{0}$ and $H_{l}$ are the hypothesis denoting absence/presence of a PU signal respectively.

Suppose total number of samples observed during a spectrum sensing time is $\mathrm{N}_{\mathrm{S}}$, average power of received signal $X(n)$ is known and noise has a constant variance $\sigma_{\mathrm{n}}{ }^{2}$. The test statistic $\mathrm{D}(\mathrm{X})$ is given as follows:

$$
D(X)=\frac{1}{N_{S}} \sum_{n=0}^{N_{S-1}} X(n)^{2}
$$


Without noise uncertainty, the central limit theorem gives performance analysis parameters $\mathrm{P}_{\mathrm{FA}}$ and $\mathrm{P}_{\mathrm{D}}$ as per [9] is given below:

$$
\begin{aligned}
& \mathrm{P}_{\mathrm{FA}}=\mathrm{Q}\left(\frac{\lambda-\sigma_{\mathrm{n}}^{2}}{\sqrt{\frac{2}{N_{S}}} \sigma_{\mathrm{n}}^{2}}\right) \\
& \mathrm{P}_{\mathrm{D}}=\mathrm{Q}\left(\frac{\lambda-\left(\mathrm{P}+\sigma_{\mathrm{n}}^{2}\right)}{\sqrt{\frac{2}{\mathrm{~N}_{\mathrm{S}}}}\left(\mathrm{P}+\sigma_{\mathrm{n}}^{2}\right)}\right)
\end{aligned}
$$

In equation (3) and (4), $\lambda$ is the detection threshold, $\mathrm{Q}($.$) is the standard Gaussian complementary function and \mathrm{SNR}=\mathrm{P} / \sigma_{\mathrm{n}}{ }^{2}$. The total error rate can be expressed as sum of error probabilities: false alarm $\left(\mathrm{P}_{\mathrm{FA}}\right)$ and Probability of missed detection $\left(\mathrm{P}_{\mathrm{MD}}\right)$ :

$$
\begin{aligned}
& P_{M D}=1-P_{D} \\
& P_{e}=P_{F A}+P_{M D}
\end{aligned}
$$

To compute the number of samples $\mathrm{N}_{\mathrm{S}}$, the detection threshold $\lambda$ is eliminated from equation (3) and (4) and expressed as:

$$
N_{S}=\frac{2\left[Q^{-1}\left(P_{F A}\right)-Q^{-1}\left(P_{D}\right)(1+S N R)\right]^{2}}{S N R^{2}}
$$

According to Equation (7), if the noise variance is completely known and constant then signal detection is possible even at low SNR just by increasing $\mathrm{N}_{\mathrm{S}}$. However, number of samples $N_{S} \rightarrow \infty$ when SNR $<<1$ (less than $0 \mathrm{~dB}$ ). In such a scenario, detection of signal becomes difficult with a fixed threshold for all SNR levels [40].

\section{A. $\quad$ With Noise Uncertainty and Fixed Threshold}

In previous section, the general model for spectrum sensing assumed constant noise with zero mean and no uncertainty. In real world, model uncertainties cannot be completely ignored since it affects the reliability of the entire system under observation. The noise uncertainty factor $\rho$ is introduced in the noise model $\rho \geq 1$ and for practical requirements the $\sigma_{\mathrm{n}}{ }^{2}$ in (3) and (4) now lie between $\left(\sigma_{n}^{2} / \rho, \rho \sigma_{n}^{2}\right)$ as presented in [9]. At low SNR and uncertain noise, the selection of threshold $(\lambda)$ by each SU becomes a crucial task to avoid missed detection or raised false alarm. In such a scenario sensing capability of the detection scheme fails. Taking noise uncertainty factor $\rho$ into account in the noise model, equation (3) and (4) are modified as follows:

$$
\begin{aligned}
& \mathrm{P}_{\mathrm{FA}}=\mathrm{Q}\left(\frac{\lambda-\rho \sigma_{\mathrm{n}}^{2}}{\sqrt{\frac{2}{N_{S}} \rho \sigma_{\mathrm{n}}^{2}}}\right) \\
& \mathrm{P}_{\mathrm{D}}=\mathrm{Q}\left(\frac{\lambda-\left(\mathrm{P}+\frac{\sigma_{\mathrm{n}}^{2}}{\rho}\right)}{\sqrt{\frac{2}{\mathrm{~N}_{\mathrm{S}}}}\left(\mathrm{P}+\frac{\sigma_{\mathrm{n}}^{2}}{\rho}\right)}\right)
\end{aligned}
$$

The uncertainty in the noise power is distributed in the interval $\left[\sigma_{\mathrm{n}}{ }^{2} / \rho, \rho \sigma_{\mathrm{n}}{ }^{2}\right]$ and can be quantified as shown in equation (10). In [27] the authors have presented the role of "SNR Wall", and its influence on detection robustness. SNR wall has been defined as the minimum required SNR for proper detection in presence of noise uncertainty. It has been explained as the margin below which the detector cannot robustly sense the presence or absence of the signal when the signal power is less than the uncertainty in noise i.e. $\mathrm{P} \leq(\rho-1 / \rho)$.

$$
S N R_{\text {wall }}=\rho-\frac{1}{\rho}
$$


It is considered that when $\rho=1$, the noise has constant power and when $\rho>1$, the noise model exhibits noise power fluctuations. While designing the sensing-error tradeoff problem, SNR wall is taken as a major constraint that leads to failure of the detection capability. At low SNR, it is assumed that the factor $(1+\mathrm{SNR}) \rightarrow 1$ and $\mathrm{N}_{\mathrm{s}}$ can be now expressed as below:

$$
N_{S}=\frac{2\left[\rho Q^{-1}\left(P_{F A}\right)-\left(\frac{1}{\rho}+S N R\right) Q^{-1}\left(P_{D}\right)\right]^{2}}{\left[S N R-\left(\frac{\rho-1}{\rho}\right)\right]^{2}}
$$

From Equation (11) it can be inferred that when SNR becomes less than the uncertainty in noise (10), then $\mathrm{N}_{\mathrm{S}} \rightarrow \infty$ and sensing failure occurs as shown in Figure 1 [28, 29].

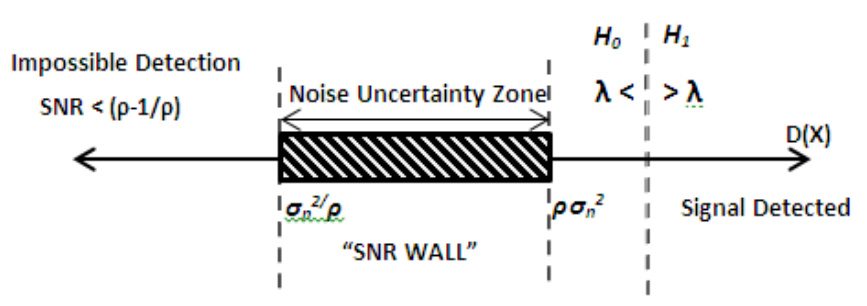

Fig 1. Testing of Hypotheses $\mathrm{H}_{0}$ and $\mathrm{H}_{1}$

Setting the threshold within the uncertainty region leads to detection failure no matter how large the sensing time is or how large is number of samples $\left(\mathrm{N}_{\mathrm{s}}\right.$ Thus, selection of detection threshold in the noise uncertain region remains a problem.

\section{B. $\quad$ With Noise Uncertainty and Dynamic Threshold}

The performance of a detection technique degrades significantly in a noise uncertain environment at very low SNR. The authors in [28] suggest the use of dynamic threshold for spectrum sensing in presence of noise uncertainty since fixed detection threshold leads to non-robust and unreliable performance. A dynamic threshold factor $\rho^{\prime}$ in the range $\left[\lambda / \rho^{\prime}, \rho^{\prime} \lambda\right]$ is introduced in detection probabilities and $\lambda$ is modified to get following equations:

$$
\begin{aligned}
& \mathrm{P}_{\mathrm{FA}}=\mathrm{Q}\left(\frac{\rho^{\prime} \lambda-\rho \sigma_{\mathrm{n}}^{2}}{\sqrt{\frac{2}{N_{S}} \rho \sigma_{\mathrm{n}}^{2}}}\right) \\
& \mathrm{P}_{\mathrm{D}}=\mathrm{Q}\left(\frac{\frac{\lambda}{\rho^{\prime}}-\left(\mathrm{P}+\frac{\sigma_{\mathrm{n}}^{2}}{\rho}\right)}{\sqrt{\frac{2}{\mathrm{~N}_{\mathrm{S}}}}\left(\mathrm{P}+\frac{\sigma_{\mathrm{n}}^{2}}{\rho}\right)}\right)
\end{aligned}
$$

Again, eliminating $\lambda$ from the equations (12) and (13), we get $\mathrm{N}_{\mathrm{S}}$ as:

$$
N_{S}=\frac{2\left[\left(\rho / \rho^{\prime}\right) Q^{-1}\left(P_{F A}\right)-\rho^{\prime}(1 / \rho+S N R) Q^{-1}\left(P_{D}\right)\right]^{2}}{\left(\rho^{\prime} S N R+\frac{\rho^{\prime}}{\rho}-\frac{\rho}{\rho^{\prime}}\right)}
$$

In Equation (14) it can be observed that if the noise uncertainty factor is equal to dynamic threshold factor numerically ( $\rho=\rho$ '), the expression will become like that without noise uncertainty as given in Equation (7). Thus, it can be interpreted that the effect of noise uncertainty can be nullified by the dynamic threshold factor $\rho$ '. But if the threshold is kept fixed then sensing might become unreliable due to uncertainty in noise at low SNR according to equation (14). 


\section{Proposed Mathematical Model}

In this section, the proposed model for spectrum sensing using dynamic double threshold concept has been presented and a new term "Threshold wall" has been coined to account for the ambiguous decision zone due to noise uncertainty at low SNR. The case when the received energy lies in the region of confusion between the two thresholds at low SNR is considered and a novel method is proposed. Furthermore, a comprehensive comparison of the proposed spectrum sensing technique with few of the existing schemes has been presented to have a better insight into the improvement in spectrum sensing performance measurement proposed by the novel method.

\section{A. $\quad$ Threshold Wall}

As discussed previously, "SNR wall" deters the selection of threshold within the noise uncertainty zone and renders unreliable performance. This may result in false alarm or missed detection and could disrupt the PU communication system. Such undesirable consequences can be prevented by dynamically adjusting the threshold according to the varying uncertainty in noise. The uncertainty in noise can be compensated by introducing dynamicity/variation in the detection threshold, expressed as below:

$$
\text { Variation in } \lambda=\left(\frac{1}{\rho^{\prime}}-\rho^{\prime}\right)
$$

From Equation (14) it can be observed that when the uncertainty in noise is at the lower limit $\left(\sigma_{\mathrm{n}}{ }^{2} / \rho\right)$, it can be balanced by dynamicity in detection threshold $\left(\rho^{\prime} \lambda\right)$. Similarly, when the noise uncertainty is at the upper limit $\left(\rho \sigma_{\mathrm{n}}^{2}\right)$, its effect can be annulled by reducing the threshold level by $(\lambda / \rho$ '). Considering the degrading effect of uncertainty in noise and the exalting repercussion of dynamic threshold factor on sensing performance, a new parameter "Threshold Wall" can be quantified through equation (16):

$$
\operatorname{THRESHOLD}_{\text {wall }}=\left(\frac{\rho^{\prime}}{\rho}-\frac{\rho}{\rho^{\prime}}\right)
$$

Threshold wall can be defined as the check parameter to keep the performance metrics within desirable limits by knowing the actual change in the detection threshold value with respect to the noise uncertainty. It can be regarded as the edge where the performance of the detector in terms of number of samples improves drastically at very low SNR. The proposed mathematical expression for threshold wall solves the problem of threshold selection in uncertain noise region. It is observed in Equation (14) $\mathrm{N}_{\mathrm{s}}$ won't tend towards infinity even at low SNR with suitable selection of the threshold wall value.

Previously, it was observed in Section II that threshold selection below the minimum required SNR ("SNR wall") leads to a situation where $\mathrm{N}_{\mathrm{s}}$ increases infinitely. It ultimately ends up in a detection failure with impractical value of probability of error $\left(P_{e} \geq 1\right)$. In such a scenario, sensing failure can be eschewed by using the threshold wall (16) for making a decision and varying $\rho$ ' in accordance with the $\rho$ when SNR is less than the uncertainty in noise. Grounded on the threshold wall, a novel sensing method based on double dynamic thresholds has been proposed in the next sub section.

\section{B. Dynamic Double Threshold}

In [31-33] two threshold based energy detection model has been proposed to mitigate the effect of noise uncertainty by maximizing the $\mathrm{P}_{\mathrm{D}}$ and minimizing the error probabilities $\left(\mathrm{P}_{\mathrm{e}}\right)$. The prediction is based on PU activity profile and switching the detection threshold dynamically between the two limits. The upper threshold to achieve higher $P_{\mathrm{D}}$ is taken as $\lambda_{\mathrm{HIGH}}$ and lower threshold for reduced $\mathrm{P}_{\mathrm{FA}}$ is considered as $\lambda_{\mathrm{LOW}}$. In this work, a novel mathematical model has been proposed for enhanced detection by considering the following conditions and illustrated in Fig. 2:

$$
\begin{aligned}
& P_{F A}=P\left(D(X)>\lambda \mid H_{0}\right) \\
& P_{D}=P\left(D(X)>\lambda \mid H_{1}\right)
\end{aligned}
$$




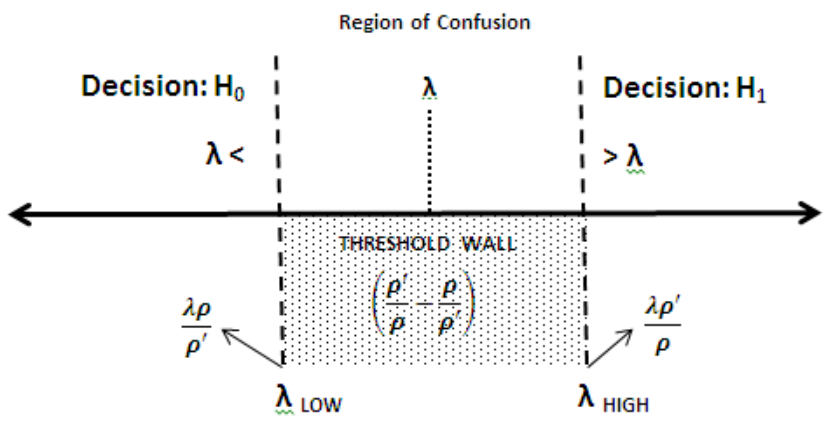

Fig 2 Proposed Dynamic Double Threshold concept for Region of Confusion

Region of confusion occurs in uncertain low SNR environment and raises the probability of missed detections or false alarm. According to the proposed technique, the detection threshold is switched between the two suggested thresholds in accordance with the variation in noise uncertainty when the detection enters the region of confusion. The concept of threshold wall as proposed in Section 3A and as proposed in [31,39] is taken as the basis for formulation of mathematical expressions of double dynamic thresholds and justified below:

$$
\begin{aligned}
& \lambda_{\text {HIGH }}=\frac{\rho^{\prime} \lambda}{\rho} \\
& \lambda_{\text {LOW }}=\frac{\rho \lambda}{\rho^{\prime}}
\end{aligned}
$$

The two thresholds expressed in equations (19) and (20) depict the two extreme points of the region of confusion. These thresholds help in achieving desired detection probability at varying levels of SNR. At low SNR the lower threshold $\lambda_{\text {LOw }}$ is considered and at higher values of SNR, the second threshold $\lambda_{\text {HIGH }}$ is taken. In both the cases, the effect of noise uncertainty $\rho$ is compensated by a nullifying effect of dynamic threshold factor $\rho$ '. Therefore, the dynamic double threshold can be varied according to the change in noise uncertainty factor at low or high SNR. This not only increases the system robustness but also reduces the probability of error. The proposed method outperforms fixed threshold detection, double threshold and cyclostationary feature detection at lower values of SNR. Furthermore, the detection threshold $\lambda$ is the mid-point of the double thresholds. The difference in the double thresholds can be computed as below:

$$
\begin{aligned}
\nabla \lambda & =\lambda_{H I G H}-\lambda_{L O W} \\
& =\frac{\rho^{\prime} \lambda}{\rho}-\frac{\rho \lambda}{\rho^{\prime}} \\
& =\lambda\left(\frac{\rho^{\prime}}{\rho}-\frac{\rho}{\rho^{\prime}}\right)
\end{aligned}
$$

In equation (21), $\left(\frac{\rho^{\prime}}{\rho}-\frac{\rho}{\rho^{\prime}}\right)$ is the detection threshold wall according to equation (16). Appropriate optimization of this factor helps in attaining desired $P_{D}$, lower $P_{e}$ and aims at finding the targeted number of samples $N_{s}$. The two novel thresholds $\lambda_{\text {HIGH }}$ and $\lambda_{\text {LOW }}$ are termed as "dynamic" as they depend on the variable uncertain noise $(\rho)$ and a dynamically changing parameter $(\rho$ '). Apart from these two factors, it is dependent on $\lambda$ which is in turn reliant on noise variance $\sigma_{n}{ }^{2}$. The proposed technique exhibits enriched detection probability when $\rho^{\prime}>\rho$. The main aim to lower the false alarm and raise detection probability at low SNR is thereby achieved. The novel double threshold-based method performs appropriately at smaller values of SNR and ensures improved sensing performance even with increasing uncertainty in noise as confirmed by the simulation results in section 4 . To validate the improvement in performance of a sensing technique with the proposed method, a comprehensive comparison of the few existing sensing methods and their parameters is given in Table I. For cyclo-stationary detection method, the test statistic is the autocorrelation coefficient assuming OFDM (Orthogonal Frequency division multiplexed) signals with $T_{d}$ as the number of data symbols; $\mathrm{T}_{\mathrm{C}}$ is the number of symbols in cyclic prefix and SNR is signal to noise ratio. The basic concept of cyclo-stationary feature detection is extraction of statistical properties of the signal. In [14] OFDM modulation is considered due its popularity and the correlation structure of the signals with cyclic prefix. Table I represents the different sensing methods, the test statistic parameter used and decision threshold. The same table and its parameters have been utilized to obtain comparative plot for different sensing methods. 


\begin{tabular}{|c|c|c|}
\hline $\begin{array}{c}\text { Sensing } \\
\text { Technique }\end{array}$ & Test Statistic D(X) & Threshold $\lambda$ \\
\hline $\begin{array}{c}\text { Energy } \\
\text { Detection } \\
\text { with Fixed } \\
\text { Threshold } \lambda \\
\text { [7] }\end{array}$ & $\mathrm{D}(\mathrm{X})=\frac{1}{\mathrm{~N}_{\mathrm{S}}} \sum_{\mathrm{n}=0}^{\mathrm{N}_{\mathrm{S}-1}} \mathrm{X}(\mathrm{n})^{2}$ & $\lambda=Q^{-1}\left(P_{F A}\right) \sigma_{n}^{2} \sqrt{\frac{2}{N_{s}}}+\sigma_{n}^{2}$ \\
\hline $\begin{array}{c}\text { Energy } \\
\text { Detection } \\
\text { with Double } \\
\text { Threshold } \lambda_{1}, \\
\lambda_{2}[28]\end{array}$ & $\mathrm{D}(\mathrm{X})=\frac{1}{\mathrm{~N}_{\mathrm{S}}} \sum_{\mathrm{n}=0}^{\mathrm{N}_{\mathrm{S}-1}} \mathrm{X}(\mathrm{n})^{2}$ & $\begin{array}{l}\lambda_{1}=(\rho-1) \lambda \\
\lambda_{2}=(\rho+1) \lambda\end{array}$ \\
\hline $\begin{array}{c}\text { Energy } \\
\text { Detection } \\
\text { with } \\
\text { Adaptive } \\
\text { Double } \\
\text { Threshold } \lambda_{1} \text {, } \\
\lambda_{2} \text { and } \lambda^{*}[30]\end{array}$ & $\mathrm{D}(\mathrm{X})=\frac{1}{\mathrm{~N}_{\mathrm{S}}} \sum_{\mathrm{n}=0}^{\mathrm{N}_{\mathrm{S}-1}} \mathrm{X}(\mathrm{n})^{2}$ & $\begin{array}{c}\lambda_{1}=(\rho-1) \lambda \\
\lambda_{2}=(\rho+1) \lambda \\
\lambda^{*}=\left(\lambda_{2}-\lambda_{1}\right) \alpha+\lambda_{1}\end{array}$ \\
\hline $\begin{array}{c}\text { Cyclo- } \\
\text { Stationary } \\
\text { Feature } \\
\text { Detection } \\
{[12]} \\
\end{array}$ & $D(X)=\frac{T_{D}}{T_{D}+T_{C}} \cdot \frac{S N R}{1+S N R}$ & $\lambda=\frac{1}{\sqrt{N_{S}}} e r f c^{-1}\left(2 P_{F A}\right)$ \\
\hline $\begin{array}{c}\text { Proposed } \\
\text { Method with } \\
\text { Double } \\
\text { Dynamic } \\
\text { Threshold }\end{array}$ & $\mathrm{D}(\mathrm{X})=\frac{1}{\mathrm{~N}_{\mathrm{S}}} \sum_{\mathrm{n}=0}^{\mathrm{N}_{\mathrm{S}-1}} \mathrm{X}(\mathrm{n})^{2}$ & $\begin{array}{l}\lambda_{H I G H}=\frac{\rho^{\prime}}{\rho} \lambda \\
\lambda_{\text {LOW }}=\frac{\rho}{\rho^{\prime}} \lambda\end{array}$ \\
\hline
\end{tabular}

Table I. Comparison of different sensing methods

\section{SIMULATION RESULTS AND DISCUSSION}

This section provides simulation results and comparative analysis to validate the proposed mathematical model in the section III. The channel is assumed to be AWGN with noise variance $\sigma_{n}^{2}=1, \mathrm{~N}_{\mathrm{S}}$ is varied between 200 to 2000 samples, SNR is set low between -20 to $10 \mathrm{~dB}$, noise uncertainty factor is taken as: $1<\rho<1.09$ and dynamic threshold factor is varied between $1<\rho^{\prime}<1.7$. The probability of false alarm is kept low at $\mathrm{P}_{\mathrm{FA}}=0.1$ and high detection probability is aimed.

At low SNR (-20 to $-2 \mathrm{~dB}$ ), when signal power is less than the uncertainty in noise $\mathrm{P}<(\rho-1 / \rho)$, then detection becomes impractical as the number of samples $\left(10 \log _{10} \mathrm{~N}_{\mathrm{S}}\right)$ increases infinitely as shown in Figure 4. It denotes the location of SNR wall with a dotted line in $\mathrm{dB}$ below which detection does not takes place for uncertainty factor of $\rho=1.002,1.02,1.04$ and 1.06 respectively. . It clearly depicts that with increasing noise uncertainty factor $(\rho>1)$, the SNR wall shifts on the higher side and makes the signal presence very vague which ultimately leads to sensing failure. The signal cannot be detected unless SNR becomes greater than the SNR wall no matter for how long the channel is sensed. The sample complexity $10 \log _{10} \mathrm{~N}_{\mathrm{s}}$ increases at low SNR and thus makes the system performance error prone. The number of samples and SNR has been expressed in dB; performance metrics are taken as $\mathrm{P}_{\mathrm{FA}}=0.01, \mathrm{P}_{\mathrm{D}}=0.9$, and $1.002<\rho<1.4$. 


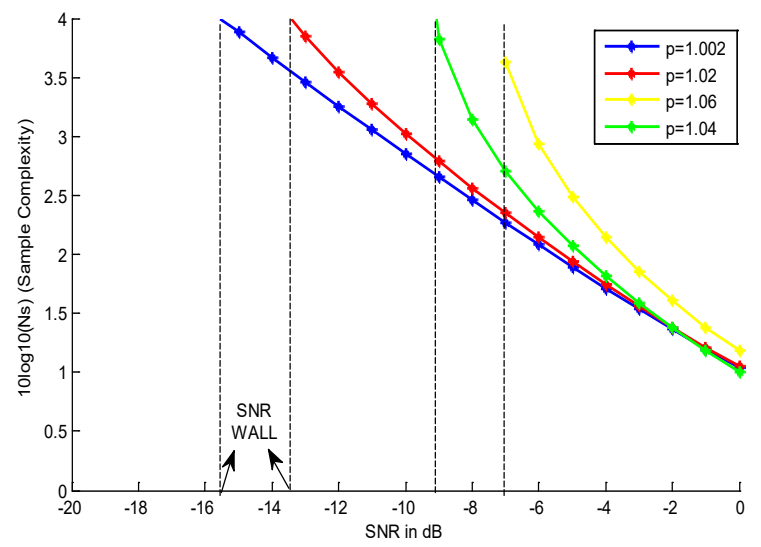

Fig. 3 Increase in $\mathrm{N}_{\mathrm{s}}$ at low SNR with increasing noise uncertainty $\rho$

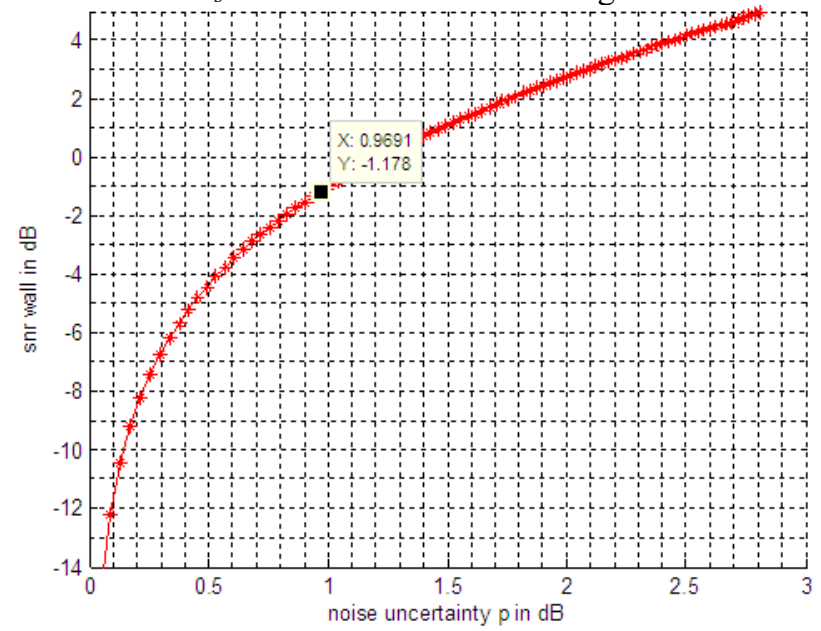

Fig. 4 "SNR Wall" as a function of noise uncertainty [25]

Figure 4 illustrates the concept of SNR wall as discussed in Section IIA. It depicts SNR as a function of noise uncertainty $\left(10 \log _{10} \rho\right)$ defined by $(10)$ and depicts the position of SNR wall for $\rho=1.02$ [24].

The effect of noise uncertainty on error rate $\left(\mathrm{P}_{\mathrm{e}}\right)$ can be seen in Figure 5. Without considering noise uncertainty, the performance of the system in terms of error rate and number of samples seems acceptable. However, error rate increases impractically beyond 1 at low SNR in presence of noise uncertainty. A significant enhancement in the sensing performance can be observed when dynamic threshold factor is introduced and increased with respect to $\rho(\rho '=1.3,1.5)$. Similarly, in Fig. 6 a graph is plotted between error rate and detection threshold in presence and absence of noise uncertainty $\rho$. It can be inferred from the plot that as dynamic threshold factor $\rho$ ' is increased in accordance with $\rho$, the error rate improves significantly thereby making the system robust. 


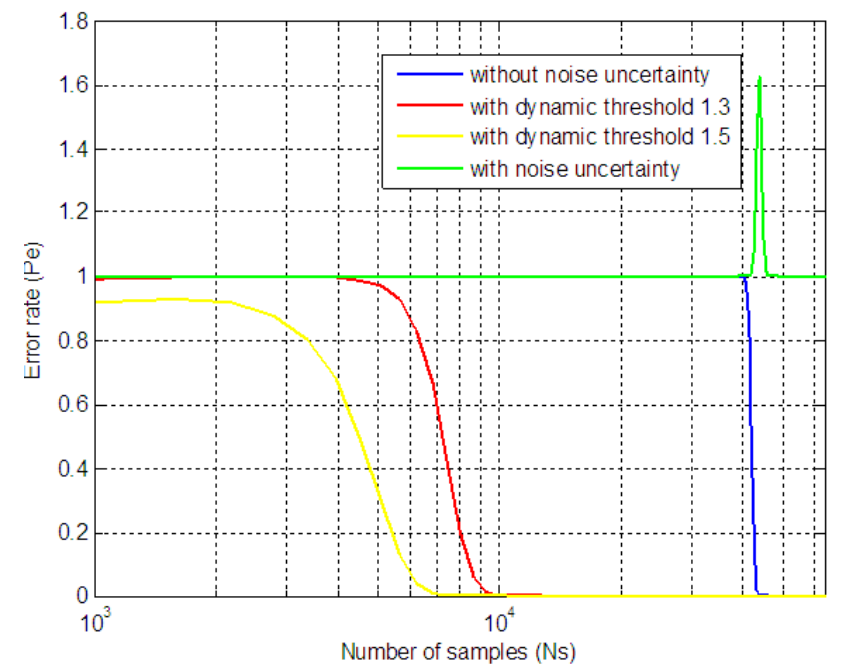

Fig. $5 \mathrm{P}_{\mathrm{e}} \mathrm{Vs} \mathrm{N}_{\mathrm{s}}$ with fixed and dynamic threshold in presence and absence of $\rho$

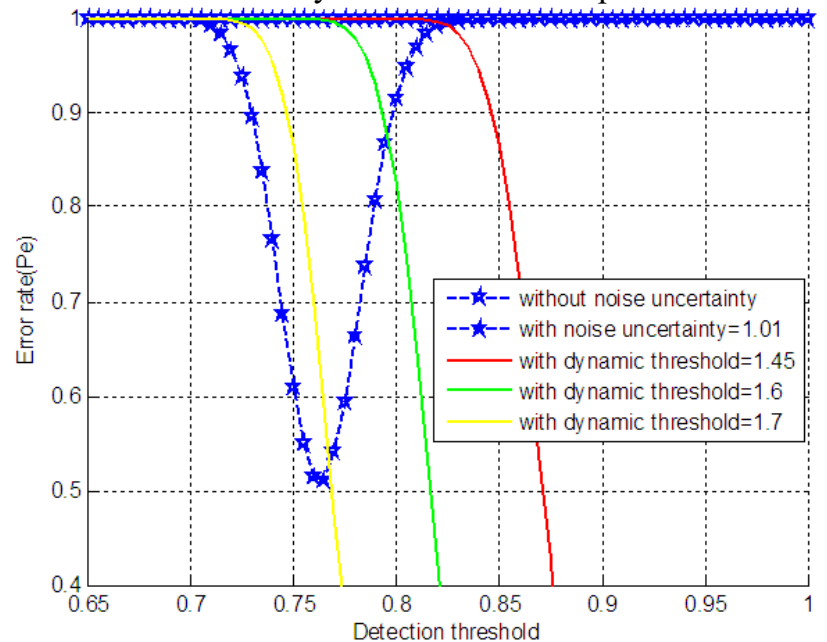

Fig. 6. $\mathrm{P}_{\mathrm{e}}$ Vs detection threshold with increasing dynamic threshold factor $\rho$ ' in presence of $\rho$

Finally, in Figure 7, the proposed model for "threshold wall" has been analyzed and validated. It demonstrates the concept of "SNR wall" for fixed threshold method and "Threshold wall" for dynamic double threshold method. The later one shows significant enhancement in sensing performance that helps in overcoming the sensing failure at low SNR in noise uncertain zone. It indicates that with proper selection of the threshold wall parameter, $\mathrm{P}_{\mathrm{e}}$ can be reduced with respect to number of samples at low SNR. 


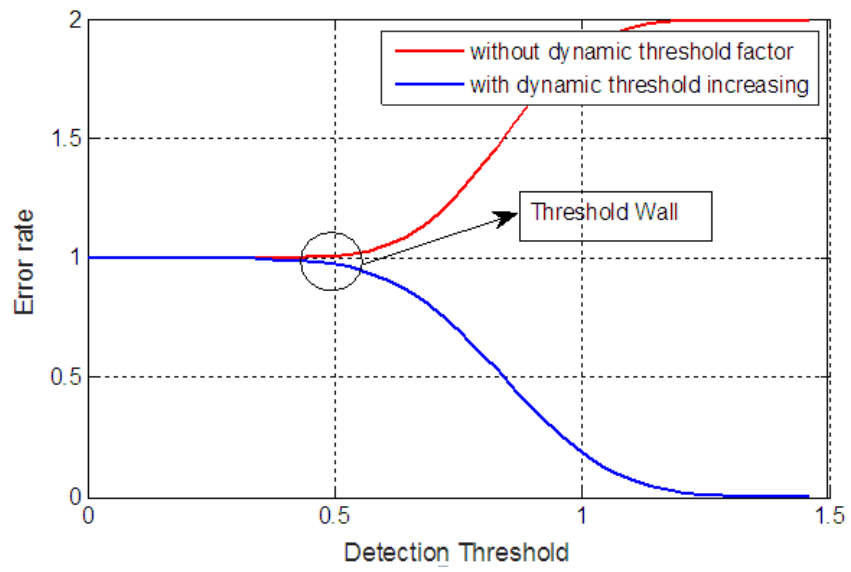

Fig. 7 "Threshold wall" to overcome detection failure

In Fig. 8, a comparative analysis of the sensing performance in terms of ROC (Receiver Operator Characteristics) curve is shown. The plot between $\mathrm{P}_{\mathrm{D}}$ and $\mathrm{P}_{\mathrm{FA}}$ signifies the enhancement in the performance of the energy detection-based spectrum sensing method using proposed dynamic double threshold as compared to few of the existing techniques mentioned in Table 1 of the previous section. It can be observed in Fig. 8 that the proposed method using dynamic threshold factor $\rho^{\prime}=1.6$ for an uncertainty in noise $\rho=1.04$, outperforms the fixed threshold, double threshold [29], adaptive double threshold [31] and cyclo-stationary feature detection [12] at $\mathrm{SNR}=-10 \mathrm{~dB}$. Thus, the proposed method exhibits robust and reliable sensing performance using dynamic double threshold.

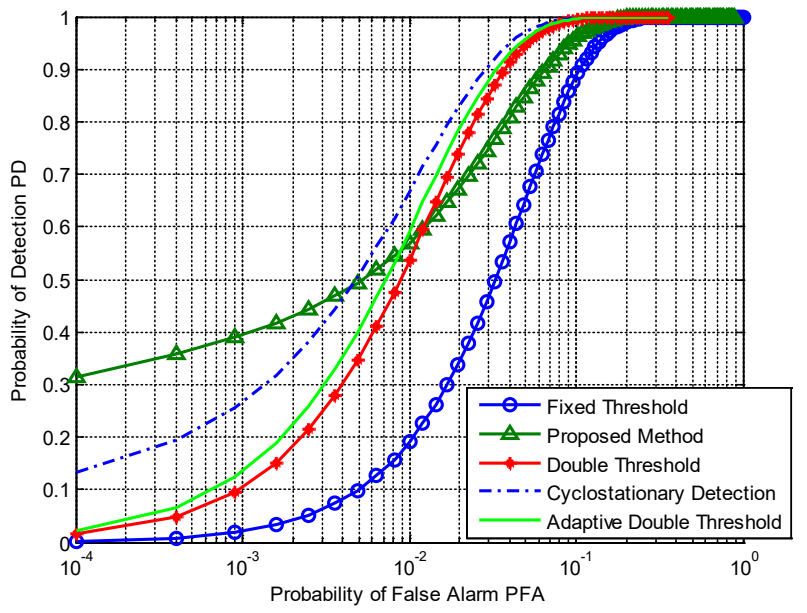

Fig. 8 Comparison of ROC for different sensing methods as per Table I.

\section{CONCLUSION}

Energy detector-based spectrum sensing technique does not provide robust and reliable performance at low SNR in presence of noise uncertainty. It leads to sensing failure and urges for a minimum SNR value over which it can perform appropriately. However, SNR wall does not provide a method to select appropriate detection threshold in uncertainty zone and results in increased error rates. A novel parameter "Threshold Wall" along with its mathematical model has been proposed that alleviates the effect of noise uncertainty by using dynamic threshold factor. The dynamicity in the threshold level with respect to noise uncertainty has been quantified as the "threshold wall" and it represents the compensation offered by the dynamic threshold factor in the sensing performance to noise uncertainty factor. To further enhance the performance the concept of dynamic double threshold is considered and a significant reduction in error rate is observed through simulation measurements by using two dynamic thresholds. The role of threshold wall for double thresholds has been analyzed based upon the newly calibrated expressions. Simulated results show that error rates decrease by increasing dynamic threshold factor with respect to noise uncertainty. The proposed dynamic double threshold concept improves the sensing performance and makes it robust at low SNR in presence of noise uncertainty. 


\section{REFERENCES}

[1] Lutu A, Perino D, Bagnulo M, Frias-Martinez E, Khangosstar J. A Characterization of the COVID-19 Pandemic Impact on a Mobile Network Operator Traffic. InProceedings of the ACM Internet Measurement Conference 2020 Oct 27 (pp. 19-33).

[2] Federal Communications Commission Spectrum Policy Task Force Report, FCC 02-155 Federal Communications Commission (Nov, 2002)

[3] M. McHenry "NSF spectrum occupancy measurements project summary" Tech. Rep. Aug. 2005.

[4] Faruk N, Bello OW, Sowande OA, Onidare SO, Muhammad MY, Ayeni AA. Large scale spectrum survey in rural and urban environments within the $50 \mathrm{MHz}-6 \mathrm{GHz}$ bands. Measurement. 2016 Sep 1;91:228-38.

[5] Federal Communications Commission Notice of Proposed Rulemaking and Order, Facilitating Opportunities for Flexible, Efficient and Reliable Spectrum Use Employing Cognitive Radio Technologies, FCC 03-322 Federal Communications Commission (Dec, 2003)

[6] Mitola J, Maguire G.Q. :Cognitive radio: making software radios more personal, IEEE Personal Communications, 6 (Aug (4)) (1999), pp. 13-18

[7] Mitola J. : Cognitive Radio: An Integrated Architecture for Software Defined Radio, Royal Institute Technology, Stockholm, Sweden (2000) (Ph.D. Diss.)

[8] Haykin, S., Thomson, D. J., Reed, J. H.: Spectrum sensing for cognitive radio, IEEE Proc., vol. 97, no. 5, pp.849-877, 2009.

[9] Urkowitz, H. :Energy detection of unknown deterministic signals, Proc. IEEE, 1967, 55(4), 523-31.

[10] Chen, Y.: Improved energy detector for random signals in Gaussian noise, IEEE Trans. Wireless Commun.,2010, 9(2), 558-63.

[11] Cabric D., Tkachenko A., Brodersen R.W. : Experimental study of spectrum sensing based on energy detection and netwotk cooperation, ACM Int. Workshop on Technology and Policy for Accessing Spectrum. Boston, USA, 2006

[12] Lunden J., Koivunen V., Huttunen A., and Poor H.V. :Collaborative Cyclo-stationary Spectrum Sensing for Cognitive Radio Systems, IEEE Transactions on Signal Processing, vol. 57, no. 11, Nov. 2009.

[13] Yan T, Xu F, Wei N, Yang Z. An Improved Cyclostationary Feature Detection Algorithm. In International Conference on Computer Engineering and Networks 2018 Aug 17 (pp. 544-555). Springer, Cham.

[14] Khan I, Singh P. Double threshold feature detector for cooperative spectrum sensing in cognitive radio networks. In2014 Annual IEEE India Conference (INDICON) 2014 Dec 11 (pp. 1-5). IEEE.

[15] Salahdine, F.; El Ghazi, H.; Kaabouch, N.; Fihri, W.F. Matched filter detection with dynamic threshold for cognitive radio networks. In Proceedings of the International Conference on Wireless Networks and Mobile Communications, Marrakech, Morocco, 20-23 October 2015; pp. 1-6.

[16] A.A. Kabeel, A.H. Hussein, A.A.M. Khalaf, H.F.A. Hamed, A Utilization of Multiple Antenna Elements for Matched Filter Based Spectrum Sensing Performance Enhancement in Cognitive Radio System, International Journal of Electronics and Communications (2019), doi: https://doi.org/10.1016/j.aeue. 2019.05.024

[17] Kumar, K.S.; Saravanan, R.; Muthaiah, R. Cognitive Radio Spectrum Sensing Algorithms based on Eigenvalue and Covariance methods. Int. J. Eng. Technol. 2013, 5, 595-601.

[18] Zeng, Y.; Liang, Y.C. Covariance based signal detections for cognitive radio. In Proceedings of the 2007 2nd IEEE International Symposium on New Frontiers in Dynamic Spectrum Access Networks, Dublin, Ireland, 17-20 April 2007; pp. 202-207.

[19] Balaji, V.; Kabra, P.; Saieesh, P.; Hota, C.; Raghurama, G. Cooperative Spectrum Sensing in Cognitive Radios Using Perceptron Learning for IEEE 802.22 WRAN. Elsevier Procedia Computing Sci. 2015, 54, 14-23.

[20] Sharma, S.K.; Lagunas, E.; Chatzinotas, S.; Ottersten, B. Application of Compressive Sensing in Cognitive Radio Communications: A Survey. IEEE Communication Survey Tutorial 2016, 18, 1838-1860.

[21] Salahdine, F.; Kaabouch, N.; El Ghazi, H. A survey on compressive sensing techniques for cognitive radio networks. J. Phys. Commun. 2016, 20, $61-73$.

[22] Arjoune Y, Kaabouch N. A comprehensive survey on spectrum sensing in cognitive radio networks: Recent advances, new challenges, and future research directions. Sensors. 2019 Jan;19 (1):126.

[23] Nguyen MT, Boveiri HR. Energy-efficient sensing in robotic networks. Measurement. 2020 Mar 10:107708.

[24] Yucek, T. \& Arslan H. : A survey of spectrum sensing algorithms for cognitive radio applications, IEEE Commun. Surveys and Tutorials, 2009, 11(1), 116-30.

[25] Abdulsattar MA, Hussein ZA. Energy detection technique for spectrum sensing in cognitive radio: a survey. International Journal of Computer Networks \& Communications. 2012 Sep 1;4(5):223.

[26] Cabric D, Mishra SM, Brodersen RW. Implementation issues in spectrum sensing for cognitive radios. In Conference Record of the Thirty-Eighth Asilomar Conference on Signals, Systems and Computers, 2004. 2004 Nov 7 (Vol. 1, pp. 772-776). Ieee.

[27] Tandra R., Sahai A.: SNR Walls for Signal Detection, IEEE 1. Sel. Topics Signal Process., Vol.2, pp. 4-17, Feb.2008.

[28] Yu G., Xi W.: A Novel Energy Detection Scheme Based on Dynamic Threshold in Cognitive Radio Systems, Journal of Computational Information Systems, Vol. 8, pp. 2245-2252, Mar. 2012.

[29] Arar, A.M., Masri, A.M., Ghannam, H.O., Tumar , I.K.: A Proposed Scheme for Dynamic Threshold Versus Noise Uncertainty in Cognitive Radio Networks (DTNU), Wireless Personal Communication Journal, Springer, Vol 96, pp 4543-4555, October 2017

[30] Xie S., Shen L: Double-threshold energy detection of spectrum sensing for cognitive radio under noise uncertainty environment, 2012 International Conference on Wireless Communications and Signal Processing (WCSP), Huangshan, 2012, pp. 1-5.

[31] Verma P, Singh B. Simulation study of double threshold energy detection method for cognitive radios. In2015 2nd International Conference on Signal Processing and Integrated Networks (SPIN) 2015 Feb 19 (pp. 232-236). IEEE

[32] Liu, F., Wang, J., \& Han, Y. : An adaptive double thresholds scheme for spectrum sensing in cognitive radio networks, IEEE international conference, (ICSPCC) (pp. 1-5), (2013).

[33] Capriglione D, Cerro G, Ferrigno L, Miele G. Performance analysis of a two-stage spectrum sensing scheme for dynamic spectrum access in TV bands. Measurement. 2019 Mar 1;135:661-71.

[34] Alkady, M.S., Abd-Elnaby, M., El-Dolil, S.A. et al. An Adaptive Sensing Time Based on SNR for Spectrum Utilization Enhancement in Cognitive Radio. Wireless Pers Commun 106, 307-319 (2019). https://doi.org/10.1007/s11277-018-6071-0

[35] Shah HA, Koo I. Reliable machine learning based spectrum sensing in cognitive radio networks. Wireless Communications and Mobile Computing. 2018 Sep 12;2018.

[36] Liu X, Zheng K, Chi K, Zhu YH. Cooperative Spectrum Sensing Optimization in Energy-Harvesting Cognitive Radio Networks. IEEE Transactions on Wireless Communications. 2020 Aug 18;19(11):7663-76. 
[37] Nasser, A., Chaitou, M., Mansour, A. et al. A Deep Neural Network Model for Hybrid Spectrum Sensing in Cognitive Radio. Wireless Pers Communications (2021). https://doi.org/10.1007/s11277-020-08013-7

[38] Mahendru, G., Shukla, A. \& Banerjee, P. A Novel Mathematical Model for Energy Detection Based Spectrum Sensing in Cognitive Radio Networks. Wireless Pers Communications 110, 1237-1249 (2020). https://doi.org/10.1007/s11277-019-06783-3

[39] Mahendru G, Shukla AK, Banerjee P, Patnaik LM. Adaptive Double Threshold Based Spectrum Sensing to Overcome Sensing Failure in Presence of Noise Uncertainty. In2019 6th International Conference on Signal Processing and Integrated Networks (SPIN) 2019 Mar 7 (pp. 466-471). IEEE.

[40] Chabbra, K., Mahendru, G., \& Banerjee, P. (2014): Effect of dynamic threshold \& noise uncertainty in energy detection spectrum sensing technique for cognitive radio systems, In International conference on signal processing and integrated networks (SPIN) (pp. 377-381). 
Figures

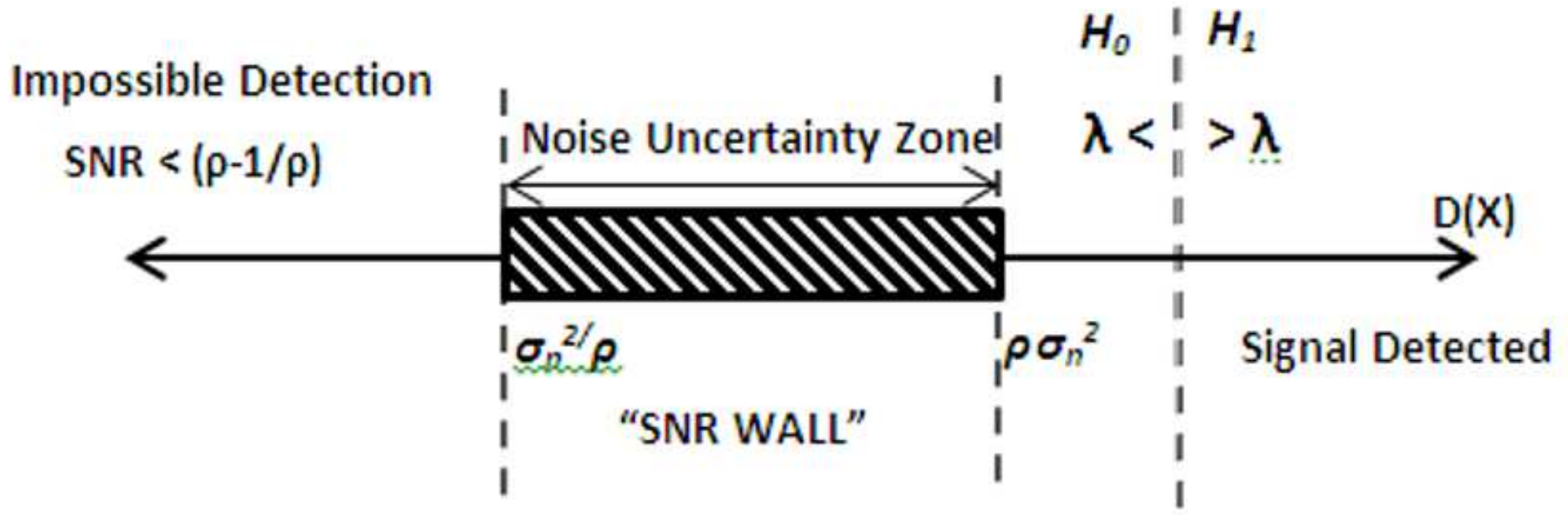

Figure 1

Testing of Hypotheses $\mathrm{HO}$ and $\mathrm{H} 1$

Region of Confusion

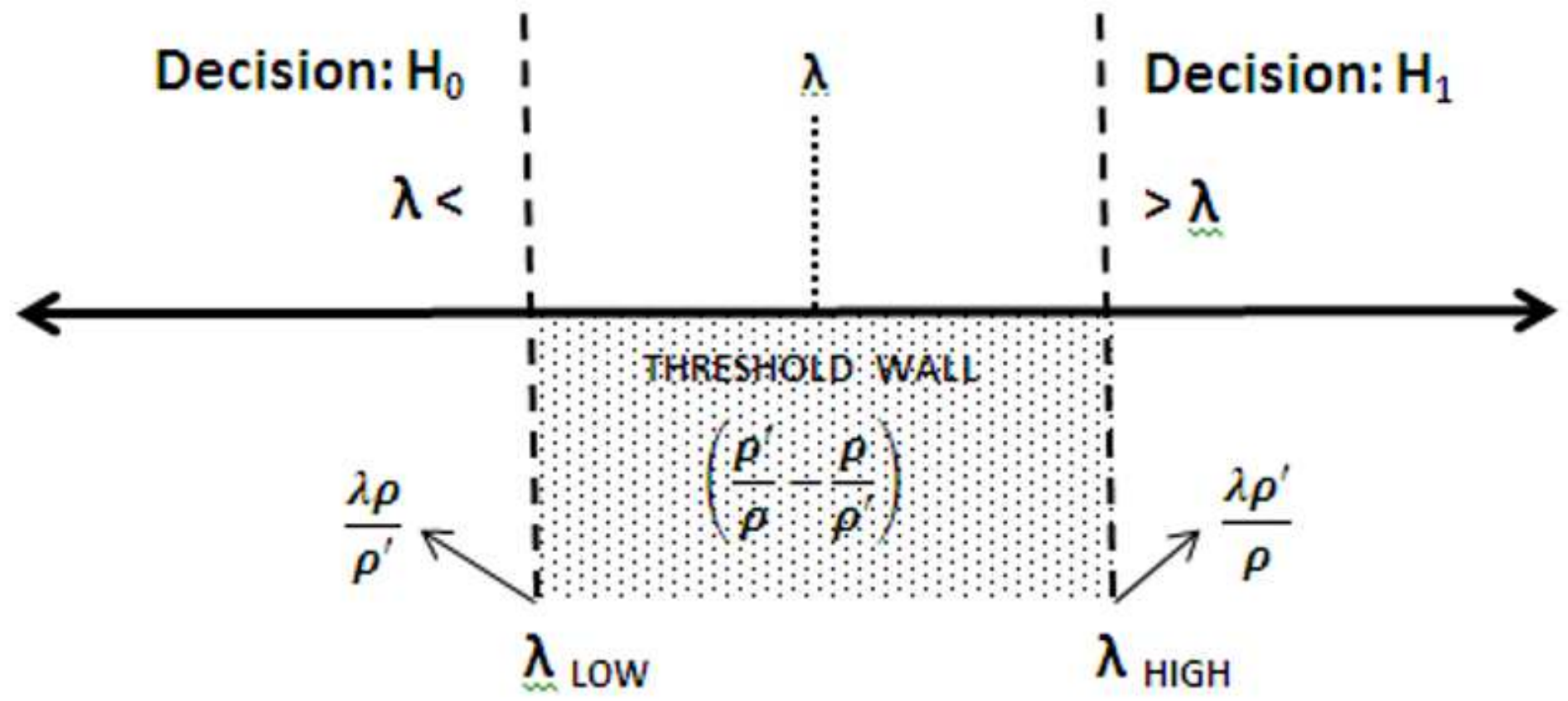

Figure 2

Proposed Dynamic Double Threshold concept for Region of Confusion 


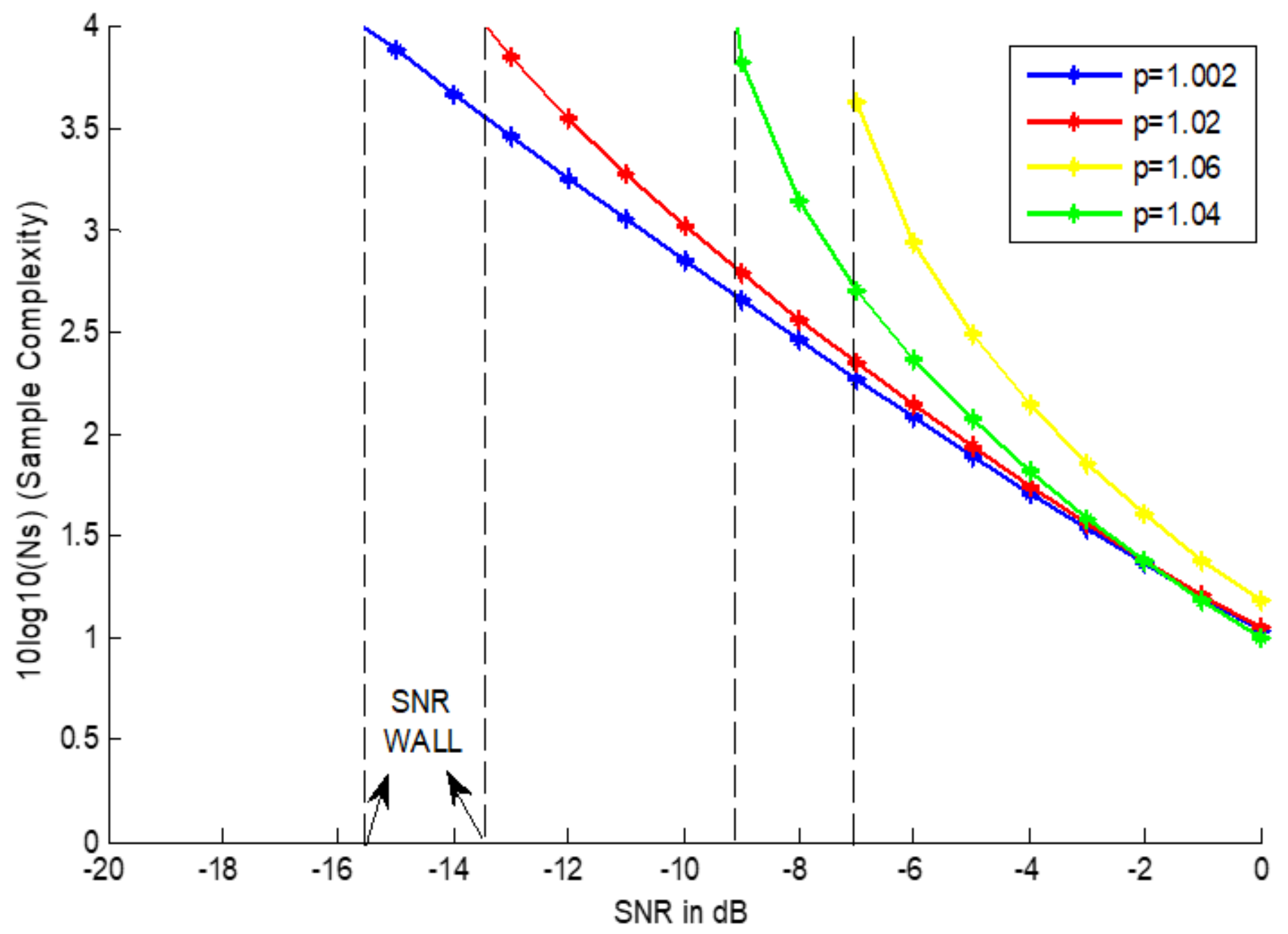

Figure 3

Increase in Ns at low SNR with increasing noise uncertainty $\rho$ 


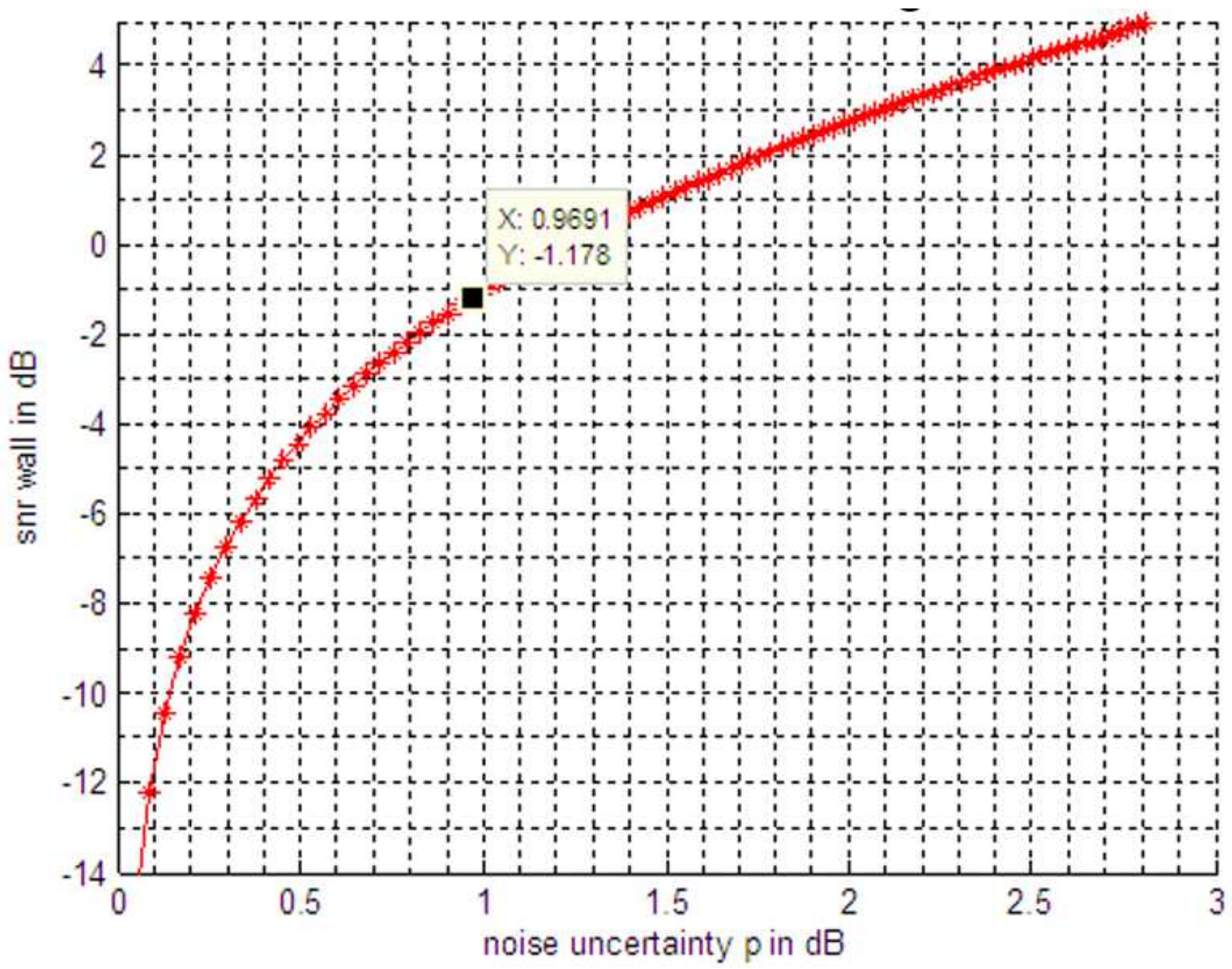

Figure 4

"SNR Wall" as a function of noise uncertainty [25] 


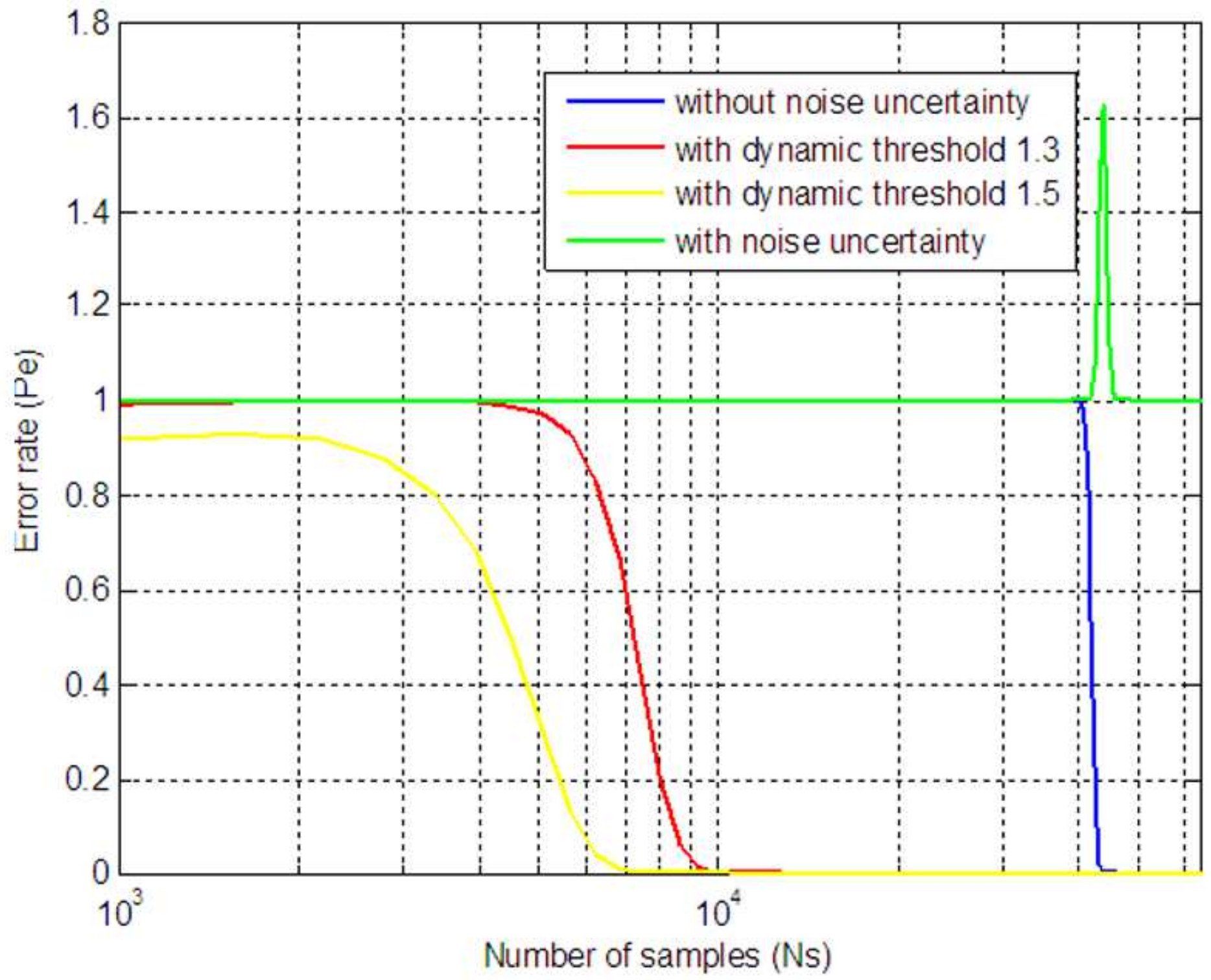

Figure 5

Pe Vs Ns with fixed and dynamic threshold in presence and absence of $\rho$ 


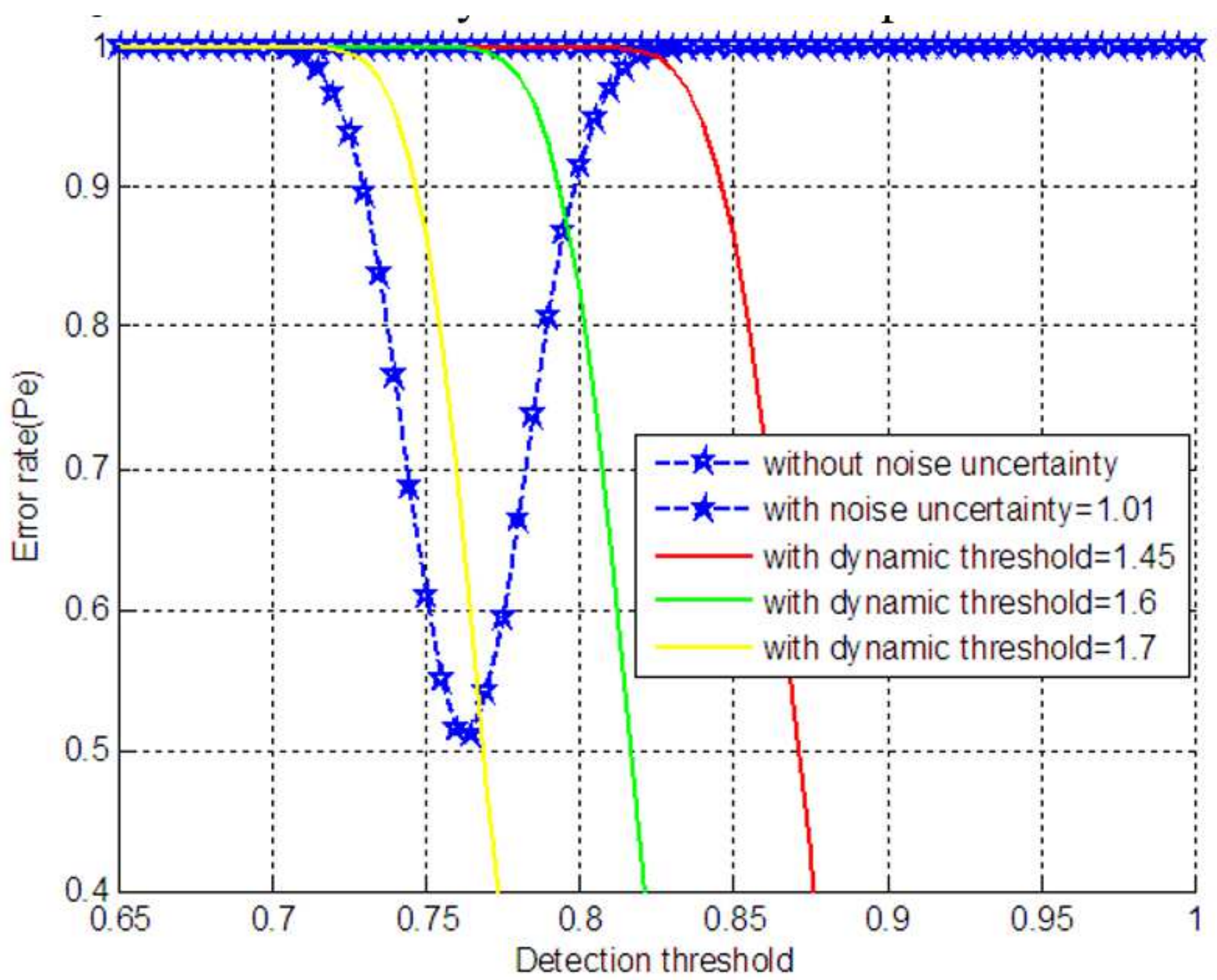

Figure 6

Pe Vs detection threshold with increasing dynamic threshold factor $\rho^{\prime}$ in presence of $\rho$ 


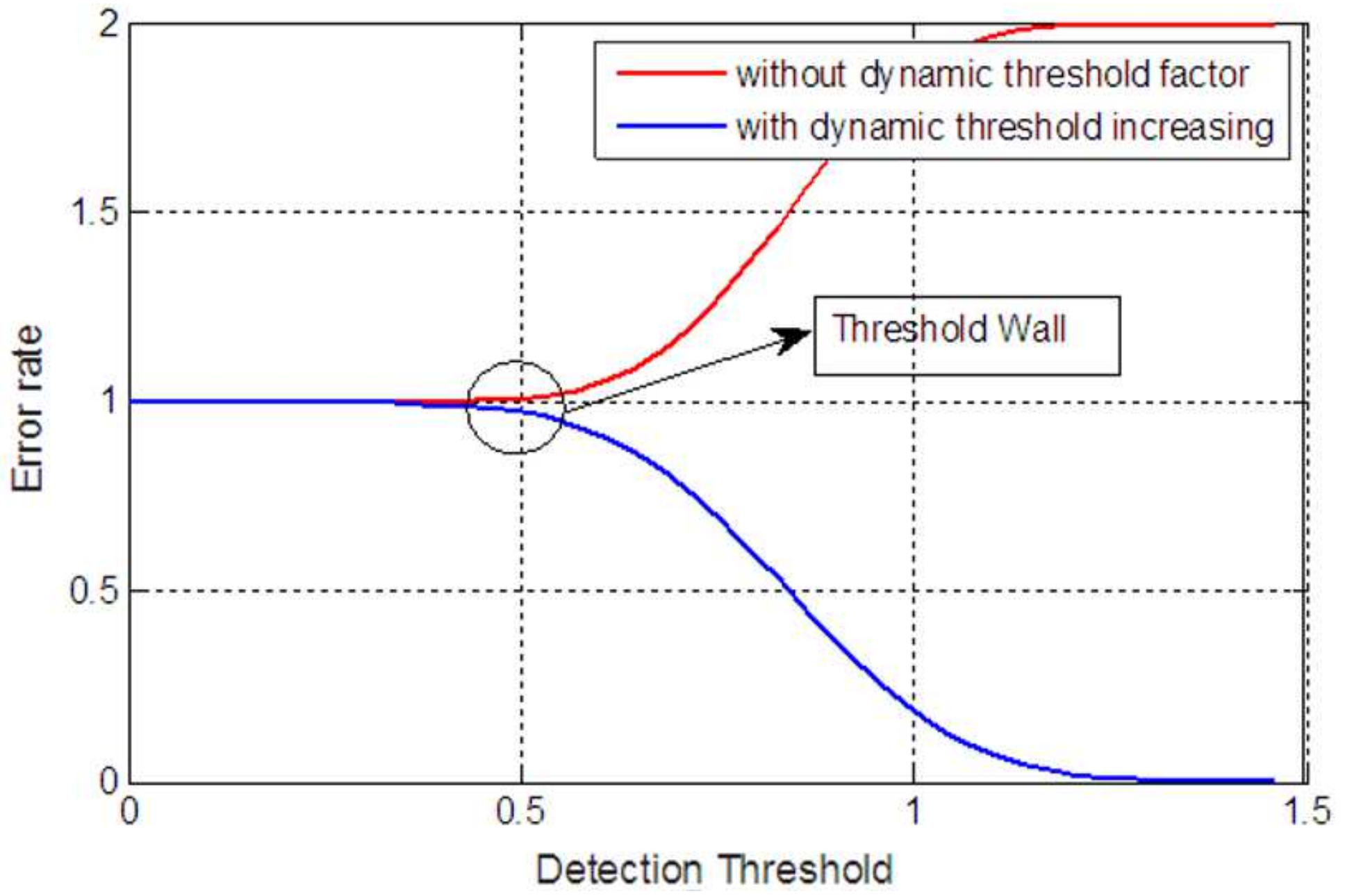

Figure 7

"Threshold wall" to overcome detection failure 


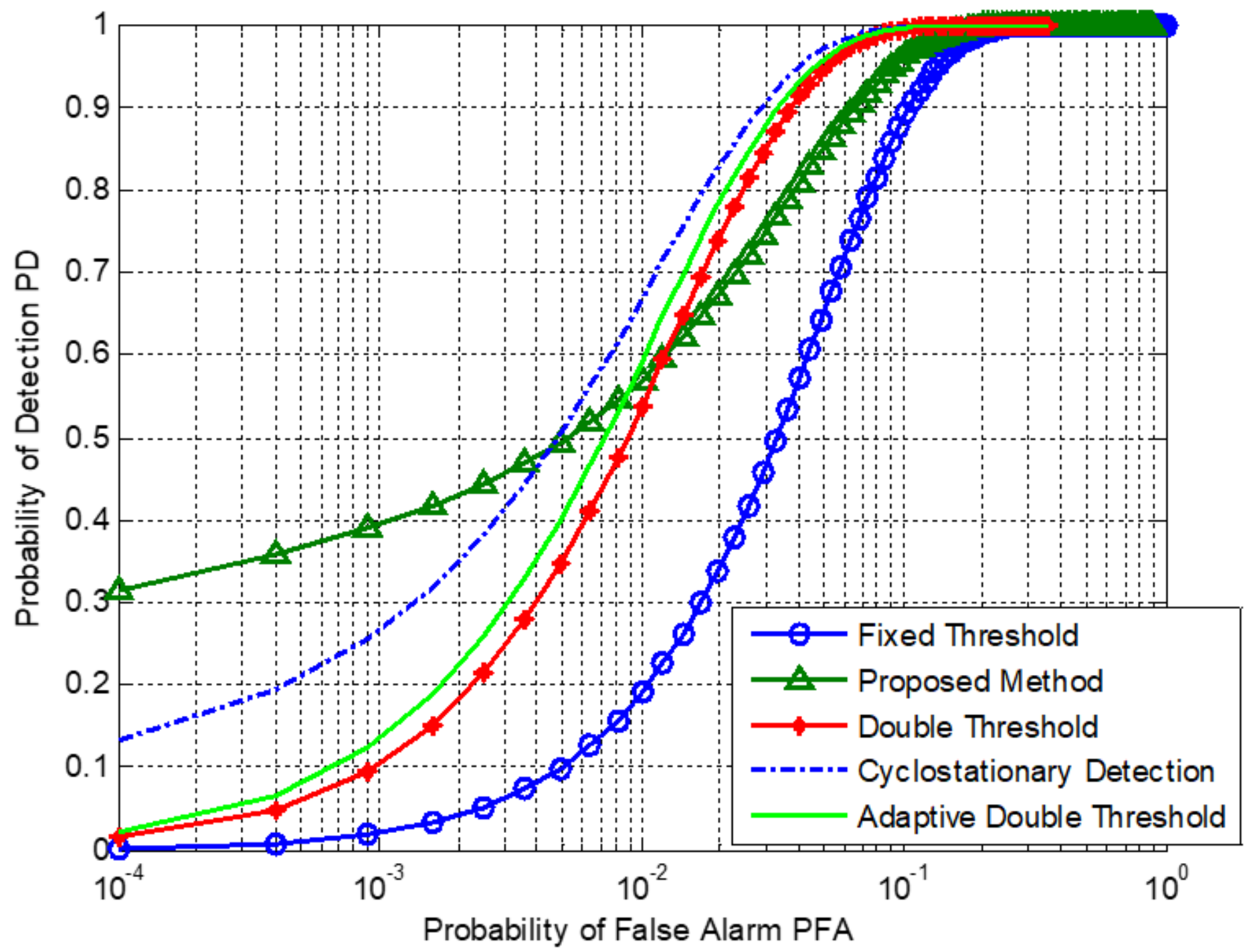

Figure 8

Comparison of ROC for different sensing methods as per Table I. 